\title{
A clinical practice providing the more appropriate airway humidification methods applied on patients with severe traumatic brain injury
}

\author{
xiaohong yin ${ }^{1}$, banyou ma ${ }^{2}$, lei yang ${ }^{2}$, hua sun $^{2}$, zhilei yun ${ }^{2}$, and yuxia wang $^{2}$ \\ ${ }^{1}$ changzhou forth people's hospital \\ ${ }^{2}$ Affiliation not available
}

May 5, 2020

\begin{abstract}
Rationale, aims and objectives: Airway humidification is an essential treatment for severe traumatic brain injury (STBI) patients after tracheotomy. To date, there was no relevant quantitative study evaluating these humidification ways and providing the appropriate method for the long-term nursing of these patients. Methods: In this study, 150 patients whom received tracheotomy treatment in our hospital from January 2016 to November 2018 were recruited into this study. Subjects were divided into three groups according to the humidification way that they received. The groups were oxygen spraying group (group A), Heat and moisture exchanger (HME) group (group B) and the heating and humidification group (group C). Phlegm viscosity, humidification effect, phlegm formation rate, daily sputum inhalation times, airway spasm, secondary lung infection, daily nursing times, nurses' internal satisfaction were evaluated. Results: The C method is superior to both A and B methods in most aspects. A method trends to happening with insufficient or excessive humidification. Phlegm scab formation is significantly less in group C. The B and $\mathrm{C}$ methods had equal humidification effects and need similar daily sputum inhalation nursing. Airway spasm was frequent happened in group A than that in groups B and C. Secondary infection happened in all groups, the number of infected patients showed a decreasing trend and the infection degree showed no difference in the first 7 days between group $\mathrm{C}$ and group A. And the severity but not the infection ratio significantly decreased in Group C on the 30th day. Method A significantly reduced nursing workload, but gained the worst humidification effects. Conclusion: Collectively, C method is more suitable for the airway nursing of patients with severe traumatic brain injury.
\end{abstract}

\section{Introduction}

Severe traumatic brain injury is often accompanied with different degrees of respiratory disorders, thus resulting in hypoxic asphyxia, elevated intracranial pressure, and these conditions further deteriorate the disease. Tracheotomy, which established an artificial airway, could maintain respiratory tract patency and is a key operation for the early treatment of severe traumatic brain injury ${ }^{1}$. During normal breathe, the upper respiratory tract will warm and wet the inhaled gas. But when an artificial airway is established, the warming and humidification effects of the patients' respiratory tract is destroyed, the dry gas can further damage the trachea and bronchial mucosa ${ }^{2}$, and the movement of respiratory cilia was affected, which causing respiratory tract blockage, pulmonary dilatation, pneumonia, posterior pituitary dysfunction and the following cognitive dysfunction ${ }^{3-5}$. Therefore, the gas must be humidified before inhaled in, that is, airway humidification, and previous study had proved that heated humidification improves clinical outcomes in children with tracheostomies ${ }^{6}$. And study recommended humidification in both invasive and noninvasive mechanical ventilation ${ }^{7}$, which suggested that humidification is essential for airway protection.

At present, there are many methods for airway humidification applied in clinical. Each method has its own disadvantage ${ }^{8-10}$, so comparative studies had been carried out, for instance, studies had compared the 
advantages of Heat and moisture exchanger (HME) and heated humidifiers (HHs) in adults and children need mechanically ventilation, though no difference was found in their study, the authors suggested further comparing of HMEs to HHs in paediatric and neonatal patients ${ }^{11}$. To date, the studies but was not sufficient, especially for some specific disease. Therefore, it is urgent to carry out systematic evaluation of some other humidification methods that frequently used in clinical, and to find out the most suitable method for airway humidification in patients with severe traumatic brain injury. In view of the fact that the common airway humidification method has been used in patients with severe traumatic brain injury in neurosurgery, and its advantages and disadvantages had also been experienced in our hospital, we chose three most respected methods in the world for comparison. We aim to select the most suitable method of airway humidification for patients with severe brain injury.

\section{Materials and methods}

\section{Design and Patients}

The project was approved by the hospital's ethical committee. All the data was collected from the record of daily nursing work after carrying out a proper screening. The information of the subjects that admitted in this study was patients who lived in the neurosurgery department with severe traumatic brain injury that need performing tracheotomy in our hospital, from January 2016 to December 2018. The patients were divided into oxygen spraying group (group A), heat and moisture exchanger (HME) (group B), and the heating and humidification group (group C) according to the humidification method the nurse chose. We excluded the patients that don't satisfy our demands. Inclusion criteria: GCS score [?] 8 points; No basic lung disease; No mechanical ventilation is required. The primary morbidity is traumatic brain injury or cerebral hemorrhage. Exclusion standard: The bleeding amount is $\& 5 \mathrm{ml}$ on the day after tracheotomy; Mechanical ventilation is still required within 3 days after tracheotomy; In-Hospital duration $i 3$ days; Death or automatic discharge; Pulmonary infection is already present. The severity was divided into two groups according to Glasgow score $(\mathrm{GCS})^{12}, 3-5$ points group and 6-8 points group. According to the in-hospital time, into and 50 patients that nursed with each method were admitted. The patients were supplied with low speed of oxygen at the first 7 days, and the patients with spontaneous respiratory were stopped oxygen supplement after they passed 7 days' acute injury stage.

\section{Regular nursing and respiratory tract humidification method}

All patients used disposable tracheotomy suits produced by Covidien llcs, and a disposable sputum suction tube was used for open sputum inhalation (Figure 1A). Sterilized injection water was chosen as uniform gas humidifying fluid, and different humidifying methods were assessed. A group: routine treatment or nursing + oxygen spraying, $20 \mathrm{ml}$ humidifying fluid was added every 2 hours and replaced every 24 hours, the oxygen spraying devices were purchased from Excellentcare Medical Ltd (Huizhou), product name: disposable medical atomizer (EM06-007), and how the devices were connected were shown in Figure 1A. Group B: routine treatment or nursing + Heat and moisture exchanger (HME), and the artificial nose were replaced every 24 hours or the sponge was contaminated. The HME device was provided by InterSurgical Company (Changzhou), the product name is: Hydro-Trach II HME with oxygen tubing. The tube was directly connected to the end of the tracheal cannula, the middle tube was connected with the oxygen tube, and the sponge at two sides was designed to collect the heat and moisture exhaled by the patients, and the devices were shown in Figure 1B. Group C: Routine treatment or nursing + heating and humidification treatment (Hamilton Medical, SFDA(I)20093540261). MR810 heating base with MR 370 heating wet tank and heating wire ventilator humidifying pipe connecting trachea sleeve, removing the other pipe loop, retaining Y-shaped joint, placing thermometer on the patient side of the pipeline, monitoring the temperature of the water mist at the entrance, Close the heated wetted tank back to the junction, the oxygen tube is connected to the small side hole of the heated wetted tank, and the sterilized injection water is connected to the oxygen tube through the adjustable infusion device to keep the liquid in the heated wetted tank within normal capacity (Figure $1 \mathrm{C}$ ). The temperature of the atomizer tank is adjusted to $39-41{ }^{\circ} \mathrm{C}$, and the temperature before entering the patient's airway is $35.3{ }^{\circ} \mathrm{C}$ according to a previous report ${ }^{13}$. The pipeline is non-polluted and not replaced. All three groups of patients were given of $3 \mathrm{~L} / \mathrm{min}$ oxygen flow, routine hemostasis, controlling 
blood pressure, reducing intracranial pressure and giving enteral nutritional support.

\section{Evaluation indicators and standards}

The following indicators were observed on $1^{\text {th }}, 3^{\text {th }}, 5^{\text {th }}$ and $7^{\text {th }}$ day after the application of the airway humidification method: 1. Phlegm viscosity (I-III): I degree:(thin phlegm) dilution such as rice soup or white foam sample, can cough up easily, after sucking sputum, there is no sputum retention on the tubing; II degree: Moderately sticky phlegm, need to be hard to cough up, after sucking phlegm, there is a small amount of sputum on the tubing; III degree: Moderately viscous, viscous appearance, often yellow, not easy to cough out, phlegm suction tube collapse due to heavy negative pressure, there is a large amount of sputum on the tubing wall and not easy to be cleaned with water. 2. Formation of phlegm scab: to observe whether the patient cough or phlegm scab when cough or suction, or the patients manifested as a rapid breathing, blood oxygen saturation or restlessness. 3. Airway spasm: sudden cough, choking, breathing difficulties, cyanosis. 4. Humidification effect: insufficient humidification: mucous liquid, less quantity, not easy to absorb, lungs breathing sound thick or dry; Good humidification: thin sputum, moderate amount, easy to absorb, clear lung breathing sound; Excessive humidification: the sputum is foamy, abundant, inexhaustible, the lungs have a large number of or rapid increased rough sounds. 5. Daily average sputum intake times. 7, Degree of secondary lung infection: three chief physicians were invited to judge the degree of lung infection according to computed tomography (CT) image and gave scores, infection degrees were divided into 1-9 points, and average score as the final score. 1-3 points set as level I, 4-6 is level IIand 7-9 is defined as level III. 8. Average nursing times: used to evaluate the number of times a nurse handles each patient each day, including daily basic care and inter-group difference of care. 9. Internal satisfaction: Evaluation of personnel satisfaction of nursing during the study.

\section{Satisfaction survey}

We use the modified version of the Minnesota Satisfaction Questionnaire (MSQ) to measure the level of satisfaction of nursing during the study ${ }^{14}$. The scale has a total of 20 topics. Topics 1-4, 7-11, 15-16 and 20 in the scale constitute the internal satisfaction and measure the satisfaction of employees with the relevant factors of the job itself. Each issue is divided into "very unsatisfactory", "unsatisfactory", "general", "satisfactory" and "very satisfactory", and is given 1-5 points, respectively.

\section{Statistical analysis}

SPSS software was used for statistical analysis, the viscosity of sputum, humidification effect, and degree of secondary lung infection were tested by rank and sum. The patients' disease distributions, phlegm scab formation, airway spasm, were analyzed using Chi square test. The patient's basic situation, daily sputum intake times, respiration related physiological indicators, daily nursing times and internal satisfaction score were analyzed with variance analysis, $\mathrm{P} ; 0.05$ was considered statistical significant.

\section{Results}

In this study, the effects of three common respiratory tract humidification methods were evaluated. There were 150 patients included in our study with the ages ranged between 18 to 70 years old. The average age, average in-hospital day, gender distribution, and GCS score distribution showed no difference among three groups (Table 1). The blood oxygen partial pressure, blood oxygen saturation and respiratory frequency showed no difference in Group B and C. The blood oxygen partial pressure and blood pressure saturation were significantly lower in Group A than those in Group B and C, and the respiratory frequency in Group A was statistically higher than that in group B and $\mathrm{C}(P ; 0.05)$ (Table 2$)$.

As Table 3 showed that, the distribution of viscosity degree of airway sputum showed that the degree of sputum viscosity showed an increasing trend during the first 7 days, suggesting that the changes might be significant in the following days. With the time of tracheotomy goes, the viscosity degree of airway sputum in group A had the fastest progressing and patients in group A was easy to form high-level viscous sputum. Table 4 indicated that the humidification effects statistically differed among three groups, $(P ; 0.05)$, group A was prone to occur insufficient or excessive humidification, and patients in group B and C gained the highest 
ratio of appropriate humidification. Method A had the least sputum intake times, while method B and C had similar more times, indicating an equal humidification effects. In group A, scab formation was significantly more than that in groups $\mathrm{B}$ and $\mathrm{C}\left(P_{j} 0.05\right)$. The incidence of airway spasm was significantly increased $\left(P_{i}\right.$ 0.05 ) in groups A than in group B and group C . After compared the degree of secondary infection in the first 7 days (Table 5), we observed that the secondary pneumonia occurred in both three groups, and the heating and humidification group showed the lowest infection ratio $(P ; 0.05)$, but the composition of the infection degree showed no difference in three groups. The secondary pneumonia on the $30^{\text {th }}$ day showed that, the infection ratio showed no difference among three groups, but the infection degree was significantly differed. The number of people with the third infection degree is significantly less in group $\mathrm{C}(P ; 0.05)$, while there was no difference was found between groups A and B.

The results (table 6) showed that the group A had the lowest average daily nursing times, as well as the nursing load, but there was no difference between groups B and C. The result of occupational satisfaction survey found that the work intensity was significantly higher in group B and C than that of group A in the inner satisfaction scale of the nurse staff $(P ; 0.05)$, and no statistical difference was found in other items (table 8).

\section{Discussion}

Patients with severe traumatic brain injury are often accompanied by different degrees of respiratory disorders. In order to prevent the deterioration of the condition, tracheotomy is often required. After tracheotomy is conducted, airway humidification must be performed, otherwise the airway mucosa will be injured and even neuron dysfunction will be brought about ${ }^{15}$. Patients with severe traumatic brain injury are often hospitalized for a long time, choosing appropriate airway humidification can effectively help severe patients reduce the occurrence of complications and reduce patient pain. In this study, we selected three relatively common humidification methods and compared their effects on airway humidification in patients with severe traumatic brain injury. Through the evaluation of the relevant indicators, we found that all three methods can effectively maintain the patient's respiratory related indicators in the normal range, and the C method has comparative advantages.

In this study, the first 7 days were chosen to evaluate these three methods, for that the clinical data in the first 7 days were relatively intact, and the patients usually transferred to better hospitals or the near-home hospitals for further therapy. In this study, we chose to give the spontaneous breathing patients with low speed of oxygen at the acute injury stage to ensure the oxygen supply of the brain, and to gain higher $\mathrm{SpO}_{2}$ and lower respiratory rate. Given the fact that $67 \%$ of the patients suffering cerebral hypoxia after severe brain injury in acute injury stage, and over oxygen supplement usually induce injury to the lung tissue, we chose 7 days as our study duration to give oxygen supplement. Some previous studies ${ }^{13,16}$ indicated that $\mathrm{C}$ method usually gains better $\mathrm{SpO}_{2}$, while our results showed that the $\mathrm{SpO}_{2}$ was lower and respiratory rate was higher in group $\mathrm{B}$ and $\mathrm{C}$ than group $\mathrm{A}$, and no difference was observed between group $\mathrm{B}$ and $\mathrm{C}$. The reason may be that, the cold air flow in group A cause the airway contraction and prevent the oxygen exchange, and the supplement of oxygen to the patients diminished the differences between group B and C.

As we know, the blood oxygen concentration will decrease when the airway spasm by cold air or the patient coughs $^{17}$, and temperature of inspired gas is closely related with the airway function ${ }^{18}$. In the $\mathrm{B}$ and $\mathrm{C}$ methods, air flow is heated and humidified, which is more comfortable and does not easily cause the patients' airway reaction. When the airway is cut, the warming and humidifying process of the upper respiratory tract to the gas disappears. Therefore, as the airway opens up, the viscosity of the phlegm in each group increases, but the $\mathrm{B}$ and $\mathrm{C}$ methods can slow this process. Compared to $\mathrm{B}$ and $\mathrm{C}$ methods, method $\mathrm{A}$ is usually gained worse humidification effects, mainly insufficient. In addition, patients with severe traumatic brain injury often need to use a large amount of dehydrating agents, thereby aggravating the dryness of the airway, which is more likely to cause viscous scab in the airway and increase the chance of lung infection in the patient ${ }^{19}$. For these reasons, the patients in group A need the least sputum intake operation, but were easy to form phlegm scab 
At present, HME is a commonly used airway humidification methods in major hospitals, mainly considering its highly effective humidification effect ${ }^{20}$. However, Boots et al. believes that HME will increase airway resistance after 24 hours of usage, so they need to be replaced every day ${ }^{21}$. A method is relatively costeffective, humidifying, but it is not stable, and it is prone to subjective judgments by nurse, thus forming excessive or insufficient humidification, and following a large amount of foam secretions, then it caused cough or shortness of breath, and reduced oxygen saturation. And the nurse needs to continuously observe the amount of humidification, which would increase the workload

$\mathrm{B}$ method is designed to use the moisture the patients exhaled to get a better humidification effect, so it can reduce airway stimulation and a good humidification effects just as $\mathrm{C}$ method, but the excreted sputum will be directly adsorbed on the filtration network. If the sponge was contaminated by the sputum, the nurse staff needs to clean the sputum adsorbed sponge frequently (about 2 hours/times), which greatly increases the workload. And when the nurses change the contaminated sponge with high frequency, the heat and moisture was released, and the patients were easy to suffer insufficient humidification and increase the viscosity of the sputum. Although the suction times of method B is less than method C, the nurses need to use extra time to deal with the sponge, and then the nursing load would not significantly be reduced.

The use of a humidifier and an unused humidifier can significantly increase the relative humidity and temperature of the air flow ${ }^{22}$, as in this study, the $\mathrm{C}$ method can provide the best humidity, temperature, and reduce airway spasm, which is conducive to the formation and discharge of sputum. The excretion of sputum is benefit for reducing the incidence of secondary pneumonia. Though the outlet tubes of the device in $\mathrm{C}$ method is not suitable for the patient's movement, it is suitable for patients with severe traumatic brain injury whom lay in bed for long time. In this study, the data were collected from our trauma center, and we focused on the humidification effects to STBI patients, but our results may not limited on the airway nursing of STBI patients, our conclusions may also serve for the other patients that need high quality of airway humidification. The shortage of this study was that, it was a retrospective study, instead of a strictly controlled randomize clinical trial, and the recruit of the patients is not randomized. In addition, we focused to evaluate the humidification effects of these three methods in the first 7 days, consecutive studies might be carried out to evaluate the long-term effects of the three methods.

\section{Conclusion}

In this study, we compared the humidification effects of three common airway humidification ways on nursing severe traumatic brain injury patients. And we found that the heating and humidification group (group C) is the most suitable method for the STBI patients.

\section{Abbreviations}

GCS: Glasgow score; CT: Computed tomography; MSQ: Minnesota satisfaction questionnaire

\section{Conflict of interest}

The authors declares no conflict of interest

\section{Ethics Approval and Consent to Participate}

The study was approved by the Hospital ethics committee.

\section{Funding Statement}

This study was supported by the Science and Technology Fund of Changzhou (WZ201528).

\section{References}

1. Lu Q, Xie Y, Qi X, Li X, Yang S, Wang Y. Is Early Tracheostomy Better for Severe Traumatic Brain Injury? A Meta-Analysis. World Neurosurg 2018;112:e324-e330. 
2. Lucato JJ, Tucci MR, Schettino GP, Adams AB, Fu C, Forti G, Jr., et al. Evaluation of resistance in 8 different heat-and-moisture exchangers: effects of saturation and flow rate/profile. Respir Care 2005;50(5):636643.

3. Pakkanen T, Nurmi J, Huhtala H, Silfvast T. Prehospital on-scene anaesthetist treating severe traumatic brain injury patients is associated with lower mortality and better neurological outcome. Scand J Trauma Resusc Emerg Med 2019;27(1):9.

4. Eshel I, Bowles AO, Ray MR. Rehabilitation of Cognitive Dysfunction Following Traumatic Brain Injury. Phys Med Rehabil Clin N Am 2019;30(1):189-206.

5. Tudor RM, Thompson CJ. Posterior pituitary dysfunction following traumatic brain injury: review. Pituitary 2018.

6. McNamara DG, Asher MI, Rubin BK, Stewart A, Byrnes CA. Heated humidification improves clinical outcomes, compared to a heat and moisture exchanger in children with tracheostomies. Respiratory care 2014;59(1):46-53.

7. American Association for Respiratory C, Restrepo RD, Walsh BK. Humidification during invasive and noninvasive mechanical ventilation: 2012. Respiratory care 2012;57(5):782-788.

8. Zhu D, Wu M, Cao Y, Lin S, Xuan N, Zhu C, et al. Heated humidification did not improve compliance of positive airway pressure and subjective daytime sleepiness in obstructive sleep apnea syndrome: A metaanalysis. PLoS ONE 2018;13(12):e0207994.

9. Nilius G, Domanski U, Schroeder M, Woehrle H, Graml A, Franke KJ. Mask humidity during CPAP: influence of ambient temperature, heated humidification and heated tubing. Nat Sci Sleep 2018;10:135-142.

10. Chikata Y, Oto J, Onodera M, Nishimura M. Humidification performance of humidifying devices for tracheostomized patients with spontaneous breathing: a bench study. Respiratory care 2013;58(9):1442-1448.

11. Gillies D, Todd DA, Foster JP, Batuwitage BT. Heat and moisture exchangers versus heated humidifiers for mechanically ventilated adults and children. The Cochrane database of systematic reviews 2017;9:CD004711.

12. Emami P, Czorlich P, Fritzsche FS, Westphal M, Rueger JM, Lefering R, et al. Impact of Glasgow Coma Scale score and pupil parameters on mortality rate and outcome in pediatric and adult severe traumatic brain injury: a retrospective, multicenter cohort study. J Neurosurg 2017;126(3):760-767.

13. Nakanishi N, Oto J, Itagaki T, Nakataki E, Onodera M, Nishimura M. Humidification Performance of Passive and Active Humidification Devices Within a Spontaneously Breathing Tracheostomized Cohort. Respiratory care 2019;64(2):130-135.

14. Olsen TW, Liao A, Robinson HS, Palejwala NV, Sprehe N. The Nine-Step Minnesota Grading System for Eyebank Eyes With Age Related Macular Degeneration: A Systematic Approach to Study Disease Stages. Invest Ophthalmol Vis Sci 2017;58(12):5497-5506.

15. Oberholzer M, Muri RM. Neurorehabilitation of Traumatic Brain Injury (TBI): A Clinical Review. Med Sci (Basel) 2019;7(3).

16. Corley A, Edwards M, Spooner AJ, Dunster KR, Anstey C, Fraser JF. High-flow oxygen via tracheostomy improves oxygenation in patients weaning from mechanical ventilation: a randomised crossover study. Intensive care medicine 2017;43(3):465-467.

17. Kaminsky DA, Lynn M. Pulmonary capillary blood volume in hyperpnea-induced bronchospasm. Am J Respir Crit Care Med 2000;162(5):1668-1673.

18. Williams R, Rankin N, Smith T, Galler D, Seakins P. Relationship between the humidity and temperature of inspired gas and the function of the airway mucosa. Critical care medicine 1996;24(11):1920-1929. 
19. Gaab M, Knoblich OE, Schupp J, Herrmann F, Fuhrmeister U, Pflughaupt KW. Effect of furosemide (lasix) on acute severe experimental cerebral edema. J Neurol 1979;220(3):185-197.

20. Boyer A, Thiery G, Lasry S, Pigne E, Salah A, de Lassence A, et al. Long-term mechanical ventilation with hygroscopic heat and moisture exchangers used for 48 hours: a prospective clinical, hygrometric, and bacteriologic study. Crit Care Med 2003;31(3):823-829.

21. Boots RJ, George N, Faoagali JL, Druery J, Dean K, Heller RF. Double-heater-wire circuits and heatand-moisture exchangers and the risk of ventilator-associated pneumonia. Crit Care Med 2006;34(3):687-693.

22. Martins De Araujo MT, Vieira SB, Vasquez EC, Fleury B. Heated humidification or face mask to prevent upper airway dryness during continuous positive airway pressure therapy. Chest 2000;117(1):142-147.
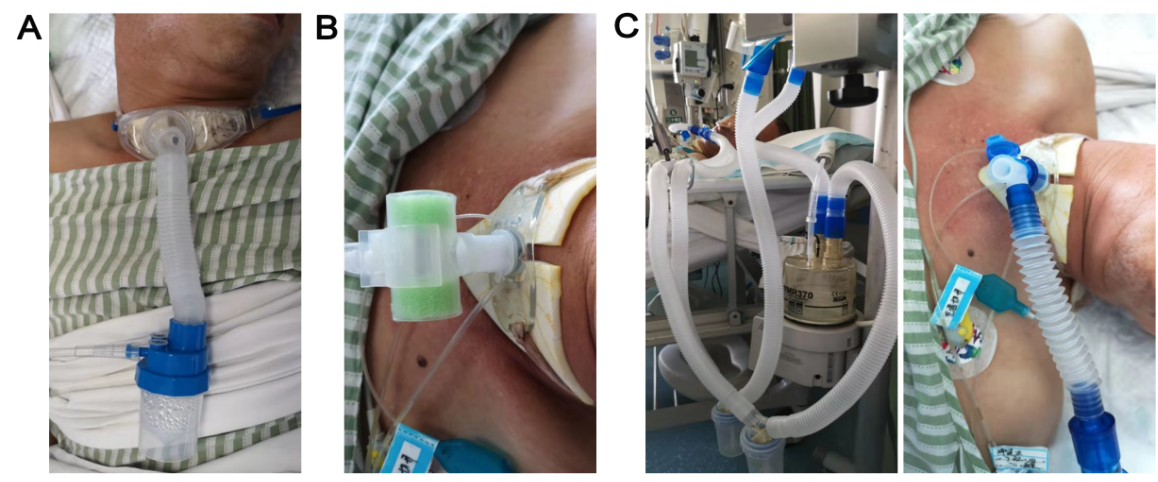

Figure 1. The images showed how the devices were connected in the three methods, (A) the oxygen spraying group (group A), (B) heat and moisture exchanger (HME), and (C) the heating and humidification group.
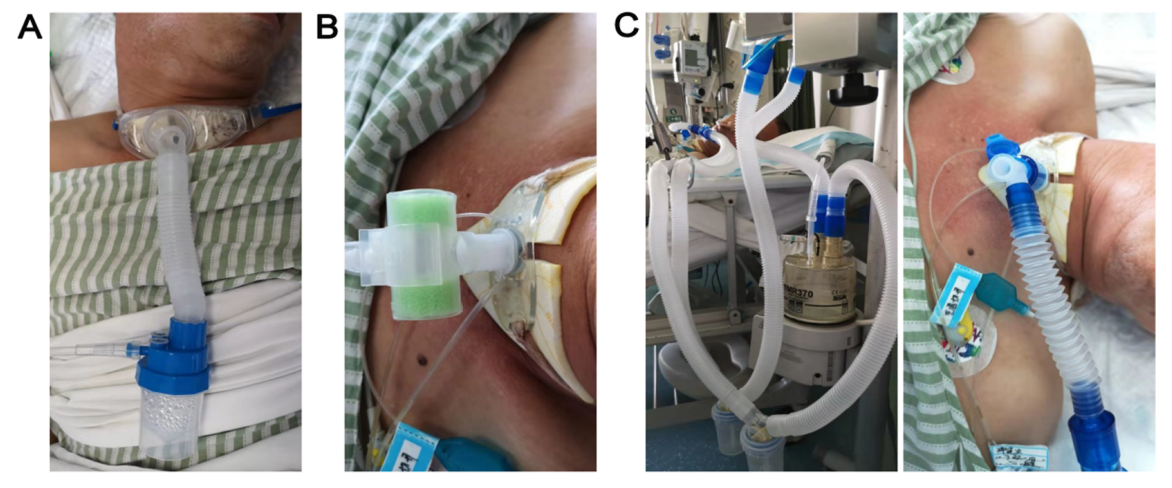

\section{Hosted file}

Table.docx available at https://authorea.com/users/295872/articles/424753-a-clinicalpractice-providing-the-more-appropriate-airway-humidification-methods-applied-onpatients-with-severe-traumatic-brain-injury 